\title{
The psychometric properties of the German version of the WHOQOL-OLD in the German population aged 60 and older
}

\author{
Ines Conrad ${ }^{1 *}$, Herbert Matschinger ${ }^{2}$, Steffi Riedel-Heller ${ }^{1}$, Carolin von Gottberg ${ }^{3}$ and Reinhold Kilian ${ }^{3}$
}

\begin{abstract}
Background: The WHOQOL-OLD is an instrument for the assessment of subjective quality of life in elderly people. It is based on the WHO definition of quality of life and is available in more than 20 languages. However, in most countries, the psychometric properties of the WHOQOL-OLD have been assessed only on the basis of small local samples and not in representative studies. In this study, the psychometric properties of the WHOQOL-OLD are evaluated based on a representative sample of Germany's elderly population.
\end{abstract}

Methods: Face-to-face interviews with 1133 respondents from the German population aged 60 years and older were conducted. Quality of life was assessed by means of the WHOQOL-BREF, the WHOQOL-OLD and the SF12. Moreover, the GDS, the DemTect and the IADL were applied for the assessment of depressive symptoms, cognitive capacities and capacity for carrying out daily activities. Psychometric properties of the WHOQOL-OLD were evaluated by means of classical and probabilistic test theory, confirmatory factor analysis and multivariate regression model.

Results: Cronbach's alpha was found to be above 0.85 for four and above .75 for two of the six facets of the WHOQOL-OLD. IRT analyses indicated that all items of the WHOQOL-OLD contribute considerably to the measurement of the associated facets. While the six-facet structure of the WHOQOL-OLD was well supported by the results of the confirmatory factor analysis, a common latent factor for the WHOQOL-OLD total scale could not be identified. Correlations with other quality of life measures and multivariate regression models with GDS, IADL and the DemTect indicate a good criterion validity of all six WHOQOL-OLD facets.

Conclusions: Study results confirm that the good psychometric properties of the WHOQOL-OLD that have been found in international studies could be replicated in a representative study of the German population. These results suggest that the WHOQOL-OLD is an instrument that is well suited to identify the needs and the wishes of an aging population.

Keywords: Quality of life, Old age, WHOQOL-OLD, Representative survey, Psychometric properties

\section{Introduction}

Given the predictions of an aging population, assessment of quality of life (QoL) of older adults is increasingly important. People in Europe are older than people in any other world region, and older adults are expected to increase to $25 \%$ of the population in several European countries by 2020 [1]. In the United States, $12 \%$ of the population or 36.3 million people are over the age of 65 years. It is projected that by $2050,21 \%$ of the American population will be over 65 [2].

\footnotetext{
* Correspondence: Ines.Conrad@medizin.uni-leipzig.de

'Institute for Social Medicine, Occupational Health and Public Health, University of Leipzig, Medical Faculty, Philipp-Rosenthal-Str. 55, 04103 Leipzig, Germany Full list of author information is available at the end of the article
}

The changing demographics have significant implications for policy makers, as well as professionals providing health and social services [3]. As a result of higher life expectancy and a trend to earlier retirement, many people in industrialized societies spend an increasing proportion of their lifetime in the "third age" [4]; that is the stage of life between retirement and the age when $50 \%$ of the age group have died [5]. Variable life courses as well as social, economic and political conditions $[4,5]$ result in a great variety of health states and living conditions.

Although QoL measurement is becoming increasingly important, issues exist regarding measurement in older adults. There is a lack of age-specific measurements [6], 
and the appropriateness of QoL instruments designed for younger adults has been questioned [6-8].

There have been a number of conceptualizations of QoL for older adults. Using Erikson's theory of life cycles $[9,10]$, we define QoL in later life as the capacity to satisfy higher order needs of Maslow's hierarchy, in particular control, autonomy, pleasure and self realization. While both approaches appropriately separate QoL from the environmental and intra-personal factors that influence it, they are limited because they ignore the subjective experience [11] and use a deductive approach to identifying the dimensions of QoL. Moreover, concepts such as control, autonomy, pleasure and self-realization may be more relevant to Western cultures. Since QoL is regarded as a universal concept reflecting the subjective experience of people [12], the individual experience, as well as cultural differences, must be taken into consideration. Consequently, the WHOQOL-OLD, an add on-module of the younger adults version of the WHOQOL for use with older adults, was developed in a cross-cultural study.

In 1991, the World Health Organization Quality of Life Project (WHOQOL) was the first attempt to take account of cultural differences during the instrument development [13-15]. This was based on the following definition of quality of life: "Quality of life is defined as individuals" perceptions of their position in life in the context of the culture and value systems in which they live and in relation to their goals, expectations, standards and concerns". The center of this definition is the subjective perception und evaluation of the living conditions by the individual. Furthermore, as a fundamental characteristic of this approach, the term quality of life is embedded into an intercultural context $[13,14,16,17]$. The intercultural comparability of the WHOQOL-OLD instrument, it was claimed, was ensured by the participation of research centers from diverse cultural areas in the development of the pilot instrument. This included the definition and operationalization of individual facets (sub-categories) and facets (main categories) of quality of life, the formulation and choice of questionnaire items, the development of response scales for single item groups and the field testing of the instrument by conducting pilot studies $[12,18]$. The result of this WHOQOL-project was the development of two generic instruments for the assessment of quality of life: the WHOQOL-100 and its short form WHOQOL-BREF [13-15,19]. Today, these two instruments are available in approximately 30 languages [19]. However, it became more and more obvious that the generic version of the WHOQOL-questionnaires was insufficient for the specific requirements of the assessment of quality of life in old age.

Therefore, a worldwide project called WHOQOL-OLD for the development of an instrument for the intercultural assessment of quality of life in old age, based on the WHOQOL-100 was initiated. Within the scope of this project, under the patronage of the $\mathrm{WHO}$, research centers from 22 countries developed an instrument for the assessment of quality of life in old age. In order to determine the dimensional structure of the quality of life concept for older people, as well as to develop facet definitions, focus groups with experts and lay persons were conducted at project baseline. The results showed that older people relate the term quality of life to social, healthrelated and environmental aspects [20]. Based on these results, items were generated whose psychometric characteristics were evaluated by a pilot study. The results of this study led to a reduction of items. The psychometric verification of the questionnaire was carried out within a survey among the respective age target-group population. The result of this study was the final version of the WHOQOLOLD questionnaire for the assessment of quality of life in older people, consisting of six new facets (Figure 1), which can be applied in combination with the WHOQOL-100 or the WHOQOL-BREF, respectively. However, the calculations of the psychometric characteristics of the final version of this instrument for the assessment of quality of life in older people (WHOQOL-OLD) were based on the same data set that was used for the development of the final version. Although the WHOQOL-OLD exists in more than 20 languages, validation of the instrument in general populations is rare. Only recently a Chinese version has been evaluated in the general population of Guangzhou (formerly Canton) [21].

In this article, the psychometric properties of the German version of the WHOQOL-OLD are assessed on the basis of a representative survey of the German population aged 60 years and older.

\section{Methods \\ Data}

In 2012, a representative, face-to-face survey of respondents 60 years and older was conducted in Germany. The sample was drawn using a random sampling procedure with three stages: (1) sample points (regional area), (2) households, and (3) individuals within the target households. Target households within 129 sample points were determined according to the random route procedure. 105 sample points comprise the area of the old and 24 the area of the new "Länder" of Germany. Target persons were selected using random digits. For the 129 sample points, a gross $\mathrm{N}$ of 5418 was chosen in order to finally realize a total sample of about 1000 respondents. In a second step for the age group $80+$, an additional sample was drawn in order to increase this part of the sample to about 300. Adding this sample of 102 respondents to the first one resulted in a total of 1133 (309 respondents aged 80 and older). 


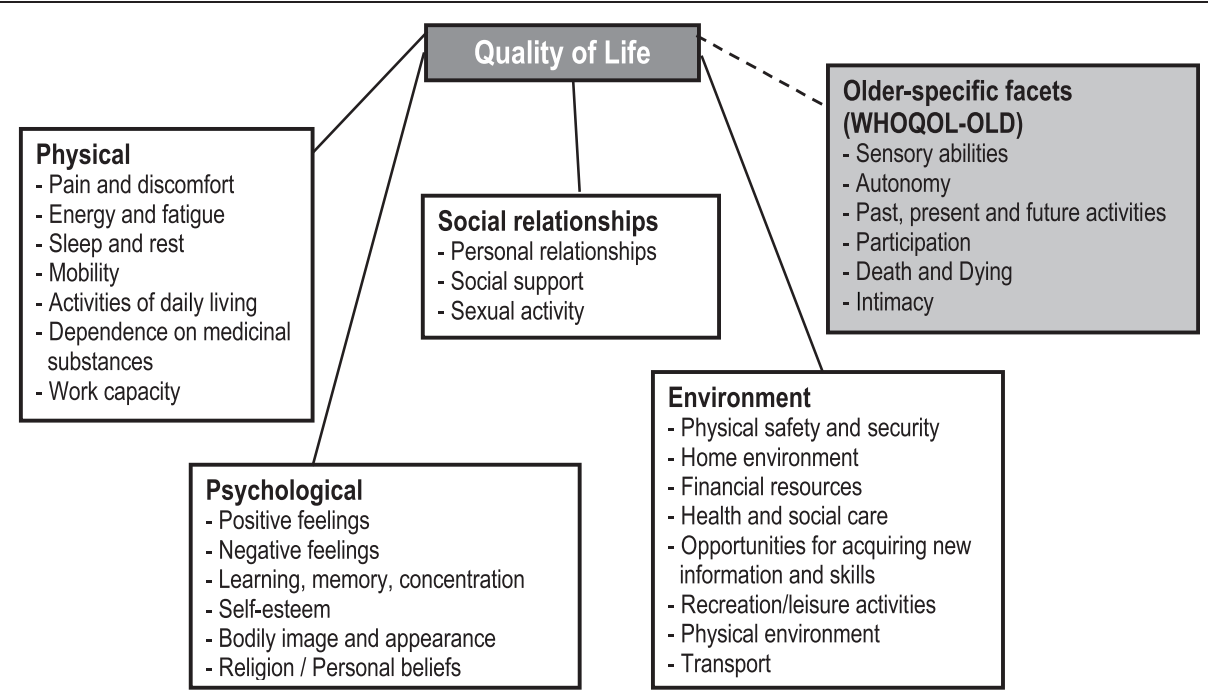

Figure 1 Dimensions of quality of life - WHOQOL-BREF \& WHOQOL-OLD (older-specific facets).

Ethical approval was obtained (University of Leipzig and Ulm University).

\section{Instruments}

To control for the inability to conduct the interview, the interview began with the DemTect in order to identify respondents with severe cognitive impairment. The DemTect is a cognitive screening test (including 5 tasks: a word list, a number transcoding task, a word fluency task, digit span reverse, delayed recall of the word list) to support the diagnosis of Mild Cognitive Impairment (MCI) and early dementia. Its transformed total score is independent of age and education [22].

Total score 13-18: Cognitive powers appropriate for subject's age

Total score 9-12: Mild cognitive impairment

Total score 0-8: Suspected dementia.

To assess subjective QoL, the German version of the WHOQOL-BREF (Figure 1) consisting of the six domains: "physical" (7 items), "psychological" (6 items), "social relationships" (3 items), "environment" (8 items) and "overall QoL" (2 items) was used [16,19]. Values of domains will be transformed into a range between 0 and 100. Internal consistency, as measured with Cronbach's alphas, of all subscales ranged between 0.57 and 0.88 . For assessing older-specific facets of quality of life, the 24-item add-on module, WHOQOL-OLD, consisting of 6 facets (sensory abilities, autonomy, past, present and future activities, social participation, death and dying and intimacy) was used (Figure 1, Table 1) [23]. Values of facets were transformed into a range between 0 and 100 , as well. Internal consistency of the subscales ranged between 0.75 for autonomy and 0.92 for intimacy.
Comorbidity was defined as the number of chronic diseases using the comorbidity list from the Federal Health Survey [24].

The respondents' functioning level concerning instrumental activities of daily living (IADL) was assessed with the Instrumental Activities of Daily Living Scale [25,26].

\section{Statistical analysis \\ Assessment of reliability}

According to recent developments in psychometric assessment in quality of life research [27-30], psychometric properties of WHOQOL-OLD were assessed by means of classical and probabilistic test theory [8].

Following the principles of the classical test theory, the reliability of the WHOQOL-OLD facets was determined on the basis of the internal consistency. Cronbach's alpha was estimated for the 6 facets of the WHOQOLOLD. The inter item correlation as well as the item scale correlation were estimated for all items in relation to the 6 facets.

To examine the reliability by means of probabilistic test theory, a Partial Credit Model (PCM) was employed [31-33]. The PCM comes from the family of IRT (Item Response Theory) models, and is an extension of the Rasch model [34,35] for polytomous items with ordered response categories:

$$
\begin{aligned}
& P\left(y_{i p}=j \mid \theta_{p}, \delta_{i l}\right)=\frac{\exp \sum_{l=0}^{j}\left(\theta_{p}-\delta_{i l}\right)}{\sum_{k=0}^{m_{i}} \exp \sum_{l=0}^{k}\left(\theta_{p}-\delta_{i l}\right)} \\
& \sum_{l=0}^{k}\left(\theta_{p}-\delta_{i l}\right)=0
\end{aligned}
$$


Table 1 The structure of the WHOQOL-OLD

\begin{tabular}{|c|c|c|}
\hline $\begin{array}{l}\text { WHOQOL-OLD } \\
\text { facet }\end{array}$ & Item no. & Item text \\
\hline \multirow{4}{*}{ I: Sensory abilities } & Old 01 & Impairments to senses affect daily life \\
\hline & Old 02 & Rate sensory functioning \\
\hline & Old 10 & $\begin{array}{l}\text { Loss of sensory abilities affect } \\
\text { participation in activities }\end{array}$ \\
\hline & Old 20 & $\begin{array}{l}\text { Problems with sensory functioning } \\
\text { affect ability to interact }\end{array}$ \\
\hline \multirow{4}{*}{ II: Autonomy } & Old 03 & Freedom to make own decisions \\
\hline & Old 04 & Feel in control of your future \\
\hline & Old 05 & Able to do things you'd like to \\
\hline & Old 11 & $\begin{array}{l}\text { People around you are respectful } \\
\text { of your freedom }\end{array}$ \\
\hline \multirow{4}{*}{$\begin{array}{l}\text { III: Past, present and } \\
\text { future activities }\end{array}$} & Old 12 & Happy with things to look forward to \\
\hline & Old 13 & $\begin{array}{l}\text { Satisfied with opportunities to } \\
\text { continue achieving }\end{array}$ \\
\hline & Old 15 & $\begin{array}{l}\text { Received the recognition you } \\
\text { deserve in life }\end{array}$ \\
\hline & Old 19 & $\begin{array}{l}\text { Satisfied with what you've achieved } \\
\text { in life }\end{array}$ \\
\hline \multirow{4}{*}{ IV: Social participation } & Old 14 & $\begin{array}{l}\text { Satisfied with the way you use } \\
\text { your time }\end{array}$ \\
\hline & Old 16 & Satisfied with level of activity \\
\hline & Old 17 & Have enough to do each day \\
\hline & Old 18 & $\begin{array}{l}\text { Satisfied with opportunity to } \\
\text { participate in community }\end{array}$ \\
\hline \multirow{4}{*}{ V: Death and dying } & Old 06 & $\begin{array}{l}\text { Concerned about the way you } \\
\text { will die }\end{array}$ \\
\hline & Old 07 & $\begin{array}{l}\text { Afraid of not being able to control } \\
\text { death }\end{array}$ \\
\hline & Old 08 & Scared of dying \\
\hline & Old 09 & Fear pain before death \\
\hline \multirow{4}{*}{ VI: Intimacy } & Old 21 & Feel a sense of companionship in life \\
\hline & Old 22 & Experience love in your life \\
\hline & Old 23 & Opportunities to love \\
\hline & Old 24 & Opportunities to be loved \\
\hline
\end{tabular}

The PCM models the probability of response category $j$ for item $i$ and person as a function of the latent "ability" $\theta_{p}$ and the threshold parameter $\delta_{i l}$ [36]. Both the thresholds and the latent ability are mapped on the same scale. The threshold parameters mark the point on the latent dimension $\theta$ where the Category Characteristic Curves intersect (e.g. the point where the probability of endorsing 2 particular adjacent categories is equal). Whether the thresholds are located on the dimension in ascending order is of major concern and not a necessary characteristic of this (ordinal) model. The PCM is suited to model sums of binary responses which are not supposed to be stochastically independent [37].
To evaluate model, two fit-indices were estimated. First, "INFIT" and "OUTFIT" which are measures for the "randomness" or "determination" of an item concerning a particular measurement model were estimated. "Values larger than 1.0 indicate unmodeled noise. Values are on a ratio scale, so that 1.2 indicates $20 \%$ excess noise. Values less than 1.0 indicate a lack of stochasticity" $[33,38-41]$. Since the INFIT is an information-weighted form of the OUTFIT which "...reduces the influence of less informative, low variance, off-target responses" [38], we expressly will focus on this parameter. This leads to the pragmatic categorization [42]:

$>2.0$ Distorts or degrades the measurement system 1.5 - 2.0 Unproductive for construction of measurement, but not degrading

0.5 - 1.5 Productive for measurement

$<0.5$ Less productive for measurement, but not degrading. May produce misleadingly good reliabilities and separations.

Secondly, the so-called Q-index (also called PersonSeparation-Index PSI) [43,44] was estimated. “The Qindex lies between zero (indicating perfect discrimination, i.e., a Guttman-pattern) and one (indicating perfect "antidiscrimination"). A value of 0.5 indicates no relationship between the individual parameter and the reaction to the item. The $Z q$ value is a transformation of the $\mathrm{Q}$-index that is approximately normally distributed if the Rasch model holds for the respective item. High positive values indicate that the item discrimination is lower than assumed by the Rasch model (under-fit), negative values indicate higher

Table 2 Sample characteristics

\begin{tabular}{lll}
\hline $\mathbf{N}$ & & $\mathbf{1 1 3 3}$ \\
\hline Age mean (SD) & & $72.5(8.7)$ \\
Gender $\mathbf{n}$ (\%) & Female & $616(54.4)$ \\
Family status $\mathbf{n}$ (\%) & Married & $559(49.3)$ \\
& $\begin{array}{l}\text { Separated, divorced, } \\
\text { widowed, never married }\end{array}$ & $574(50.7)$ \\
Living arrangement (\%) & Alone & $483(42.6)$ \\
& With others & $654(57.4)$ \\
Education (\%) & High & $479(42.3)$ \\
& Basic & $654(57.7)$ \\
DemTect categories $\mathbf{n}$ (\%) & 0 severe impairments & $104(9.5)$ \\
& 1 mild impairments & $269(24.3)$ \\
& 2 no impairments & $730(66.3)$ \\
Number of chronic & & $5.3(3.8)$ \\
diseases mean (SD) & & $6.7(1.7)$ \\
IADL mean (SD) & & $3.4(3.8)$ \\
\hline GDS mean (SD) & &
\end{tabular}


discrimination than assumed (over-fit)" [45]. ZQ values within the range of -1.96 and 1.96 indicate that the Qindex of an item is in the expected range with a probability of $95 \%$.

The thresholds for the answering categories and the distributions of the latent scale dimensions are presented in the person-item maps (PIM). The histograms in the upper part of the PIM represent the distribution on the latent scale of each facet. The lines in the lower part of the PIM represent the ranges of the latent scales with the means symbolized, as dark dots and the thresholds of the k-1 answering categories symbolized as circles

Table 3 Reliability parameters of the WHOQOL-OLD facets

\begin{tabular}{|c|c|c|c|c|c|c|}
\hline \multirow[b]{2}{*}{$\begin{array}{l}\text { Item no./WHOQOL-OLD } \\
\text { facet }\end{array}$} & \multicolumn{4}{|c|}{ Classical test theory } & \multicolumn{2}{|l|}{ IRT } \\
\hline & $\begin{array}{l}\text { Item-test } \\
\text { correlation }\end{array}$ & $\begin{array}{l}\text { Corrected item-test } \\
\text { correlation }\end{array}$ & $\begin{array}{l}\text { Inter-item } \\
\text { correlation }\end{array}$ & $\begin{array}{l}\text { Cronbach's } \\
\text { alpha if item } \\
\text { deleted/alpha }\end{array}$ & Q-Index & INFIT \\
\hline Old 01 & 0.8882 & 0.7849 & 0.6350 & 0.8369 & -0.1828 & 0.687 \\
\hline Old 02 & 0.9137 & 0.8337 & 0.6035 & 0.8167 & -0.5315 & 0.509 \\
\hline Old 10 & 0.8206 & 0.6755 & 0.7097 & 0.8793 & 0.6041 & 1.031 \\
\hline Old 20 & 0.8255 & 0.7114 & 0.6842 & 0.8669 & 0.1172 & 0.780 \\
\hline \multirow[t]{2}{*}{ Sensory abilities } & & & 0.6581 & 0.8842 & \multicolumn{2}{|c|}{ Andrich reliability } \\
\hline & & & & & \multicolumn{2}{|l|}{0.798} \\
\hline Old 03 & 0.7790 & 0.6062 & 0.4057 & 0.6696 & -0.5757 & 0.662 \\
\hline Old 04 & 0.7595 & 0.5148 & 0.4694 & 0.7218 & 0.5710 & 0.847 \\
\hline Old 05 & 0.7472 & 0.5558 & 0.4371 & 0.6949 & -0.0180 & 0.747 \\
\hline Old 11 & 0.7594 & 0.5409 & 0.4519 & 0.7017 & 0.1018 & 0.797 \\
\hline \multirow[t]{2}{*}{ Autonomy } & & & 0.4015 & 0.7537 & \multicolumn{2}{|c|}{ Andrich reliability } \\
\hline & & & & & \multicolumn{2}{|l|}{0.703} \\
\hline Old 12 & 0.8197 & 0.6062 & 0.4228 & 0.6851 & -0.1279 & 0.694 \\
\hline Old 13 & 0.7849 & 0.5811 & 0.4377 & 0.6945 & -0.0293 & 0.737 \\
\hline Old 15 & 0.7141 & 0.5254 & 0.4750 & 0.7263 & 0.2049 & 0.804 \\
\hline Old 19 & 0.7402 & 0.5551 & 0.4608 & 0.7112 & -0.0690 & 0.771 \\
\hline \multirow[t]{2}{*}{ Past, present and future activities } & & & 0.4491 & 0.7619 & \multicolumn{2}{|c|}{ Andrich reliability } \\
\hline & & & & & \multicolumn{2}{|l|}{0.751} \\
\hline Old 14 & 0.7514 & 0.5667 & 0.6817 & 0.8596 & 1.3272 & 1.071 \\
\hline Old 16 & 0.8309 & 0.7167 & 0.5781 & 0.8042 & -0.4557 & 0.694 \\
\hline Old 17 & 0.8897 & 0.7891 & 0.5318 & 0.7656 & -0.9818 & 0.540 \\
\hline Old 18 & 0.8600 & 0.7146 & 0.5804 & 0.8019 & 0.0255 & 0.718 \\
\hline \multirow[t]{2}{*}{ Social participation } & & & 0.5930 & 0.8502 & \multicolumn{2}{|c|}{ Andrich reliability } \\
\hline & & & & & \multicolumn{2}{|l|}{0.801} \\
\hline Old 06 & 0.8443 & 0.7211 & 0.5859 & 0.8095 & -0.5266 & 0.737 \\
\hline Old 07 & 0.8806 & 0.7666 & 0.5559 & 0.7884 & -1.2724 & 0.610 \\
\hline Old 08 & 0.8163 & 0.6747 & 0.6163 & 0.8280 & 0.4899 & 0.849 \\
\hline Old 09 & 0.8044 & 0.6432 & 0.6403 & 0.8415 & 1.1852 & 0.946 \\
\hline \multirow[t]{2}{*}{ Death and dying } & & & 0.5996 & 0.8567 & \multicolumn{2}{|c|}{ Andrich reliability } \\
\hline & & & & & \multicolumn{2}{|l|}{0.829} \\
\hline Old 21 & 0.8675 & 0.7667 & 0.7625 & 0.9051 & 0.5563 & 0.901 \\
\hline Old 22 & 0.9230 & 0.8517 & 0.7022 & 0.8767 & -0.5745 & 0.578 \\
\hline Old 23 & 0.8958 & 0.8153 & 0.7278 & 0.8891 & -0.1093 & 0.706 \\
\hline Old 24 & 0.8897 & 0.8018 & 0.7369 & 0.8934 & 0.1258 & 0.765 \\
\hline \multirow[t]{2}{*}{ Intimacy } & & & 0.7324 & 0.9162 & \multicolumn{2}{|c|}{ Andrich reliability } \\
\hline & & & & & \multicolumn{2}{|l|}{0.888} \\
\hline
\end{tabular}




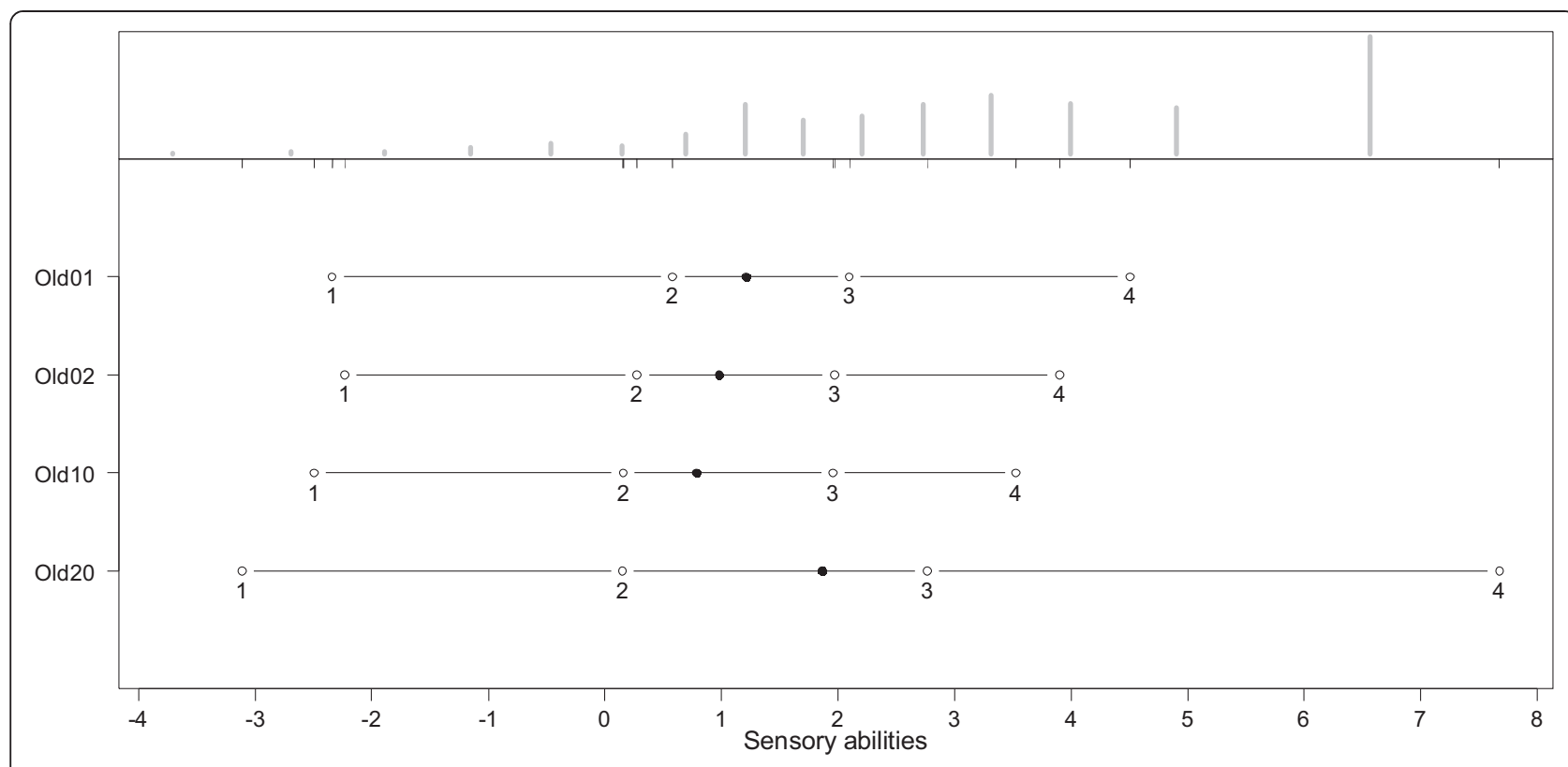

Figure 2 Person-item map (PIM) of the WHOQOL-OLD facet "sensory abilities".

with the number of the category. As an indicator of a high reliability, all thresholds should have the same ascending order. The discriminatory power of the items is represented by the range between the thresholds. Small ranges represent a high discriminatory power and vice versa. Since the PCM supposes an ordinal scaling model, it does not require equal ranges between thresholds.

\section{Assessment of validity}

The construct validity of the WHOQOL-OLD was assessed by means of a first order and second order confirmatory factor analysis. The first model represents the 6 factor structure in the sense of a congeneric measurement model [46]. The second model contains an additional factor of $2^{\text {nd }}$ order that was included to investigate whether

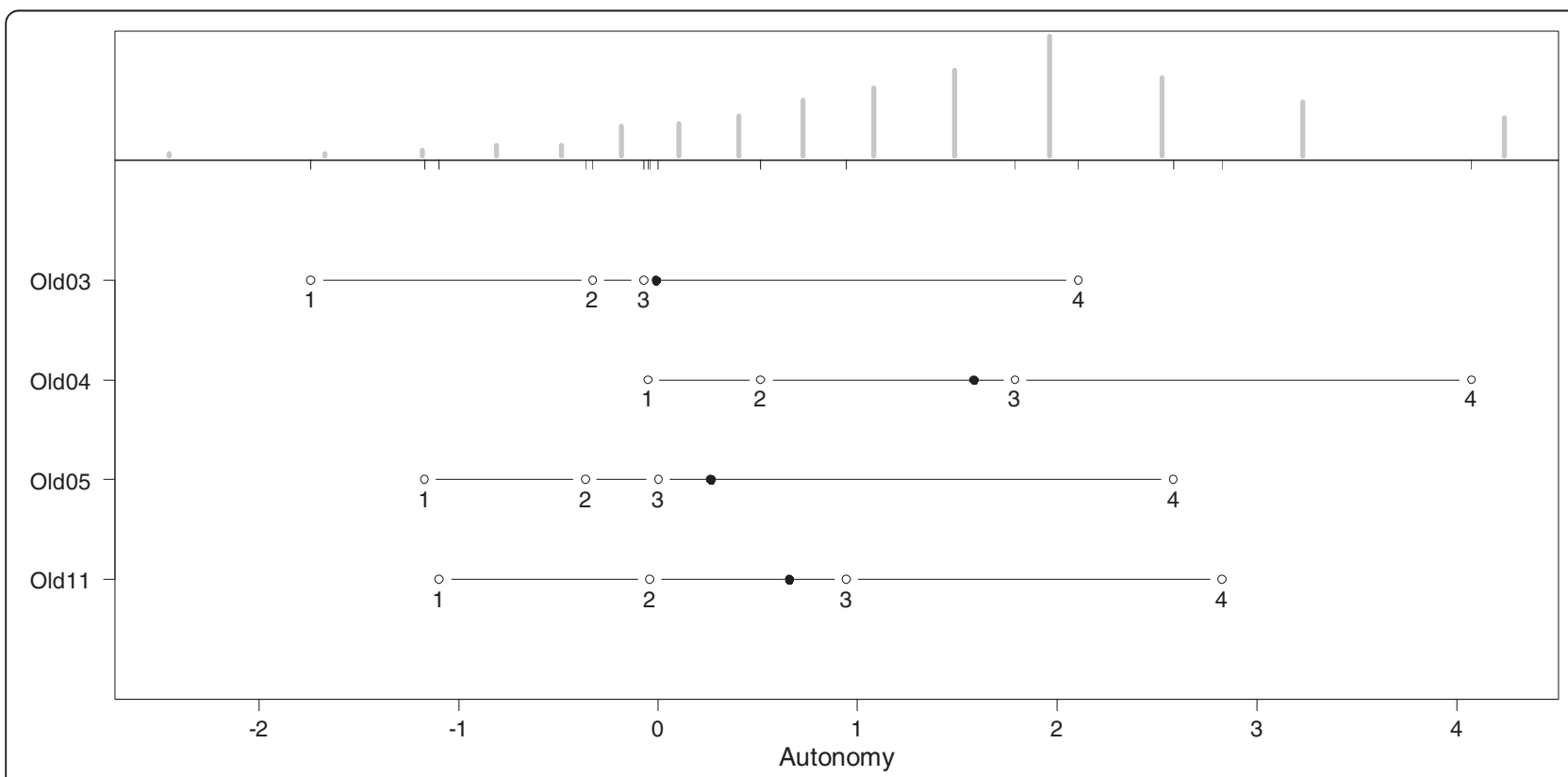

Figure 3 Person-item map (PIM) of the WHOQOL-OLD facet "autonomy". 


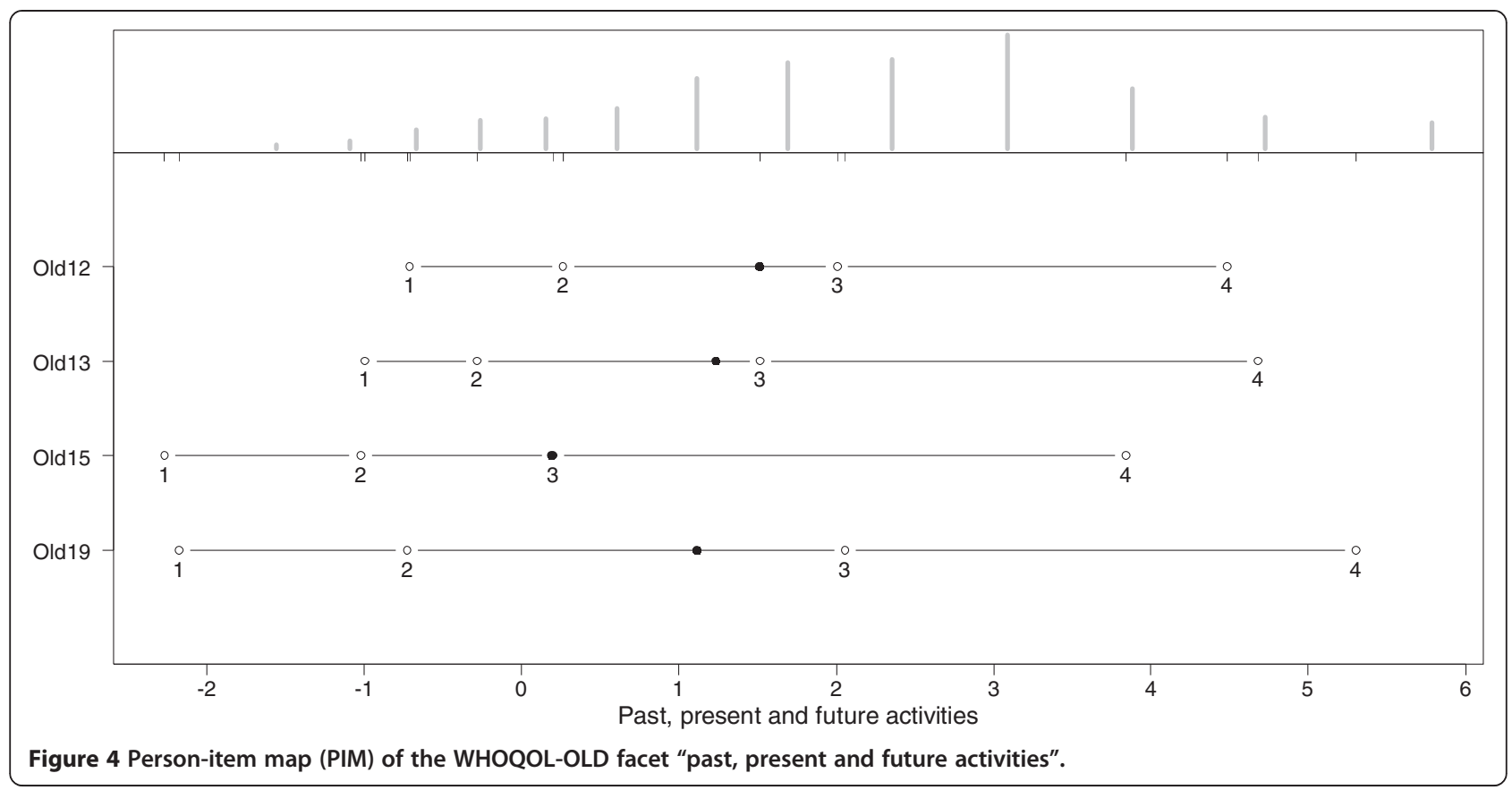

the construct "Quality of Life" might be represented by one single dimension.

Convergent validity of the WHOQOL-OLD is determined by examining the correlations between the WHOQOL-OLD facets and a set of criterion variables. Criterion variables include generic quality of life measured by the WHOQOL-BREF and the SF12 Physical Health Index (SF12 PHI) and the SF12 Mental Health Index (SF12 MHI).

Discriminant validity was assessed by multivariate regression models for each of the WHOQOL-OLD facets with the socio-demographic characteristics, the living situation, the GDS, the IADL, the number of chronic diseases and the cognitive status measured by the DemTect as independent variables.

\section{Software}

The CFA was estimated by Mplus 7.11 [47]. Analyses for the PCM were conducted by the package $\mathbf{e R m}$ [48] or ltm [49] for R. The Q-Index was computed using WINMIRA [45]. The indices regarding "classical test

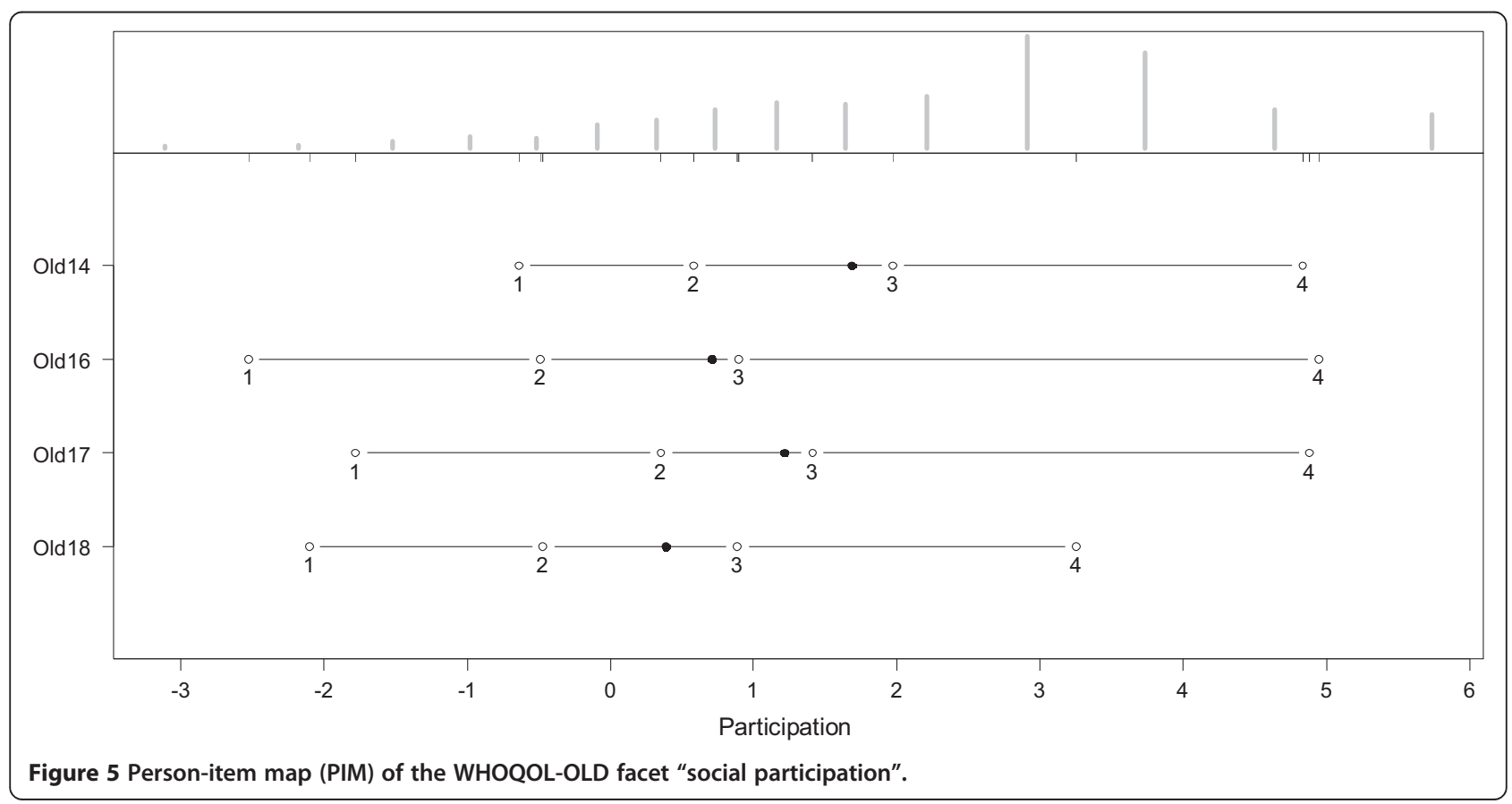




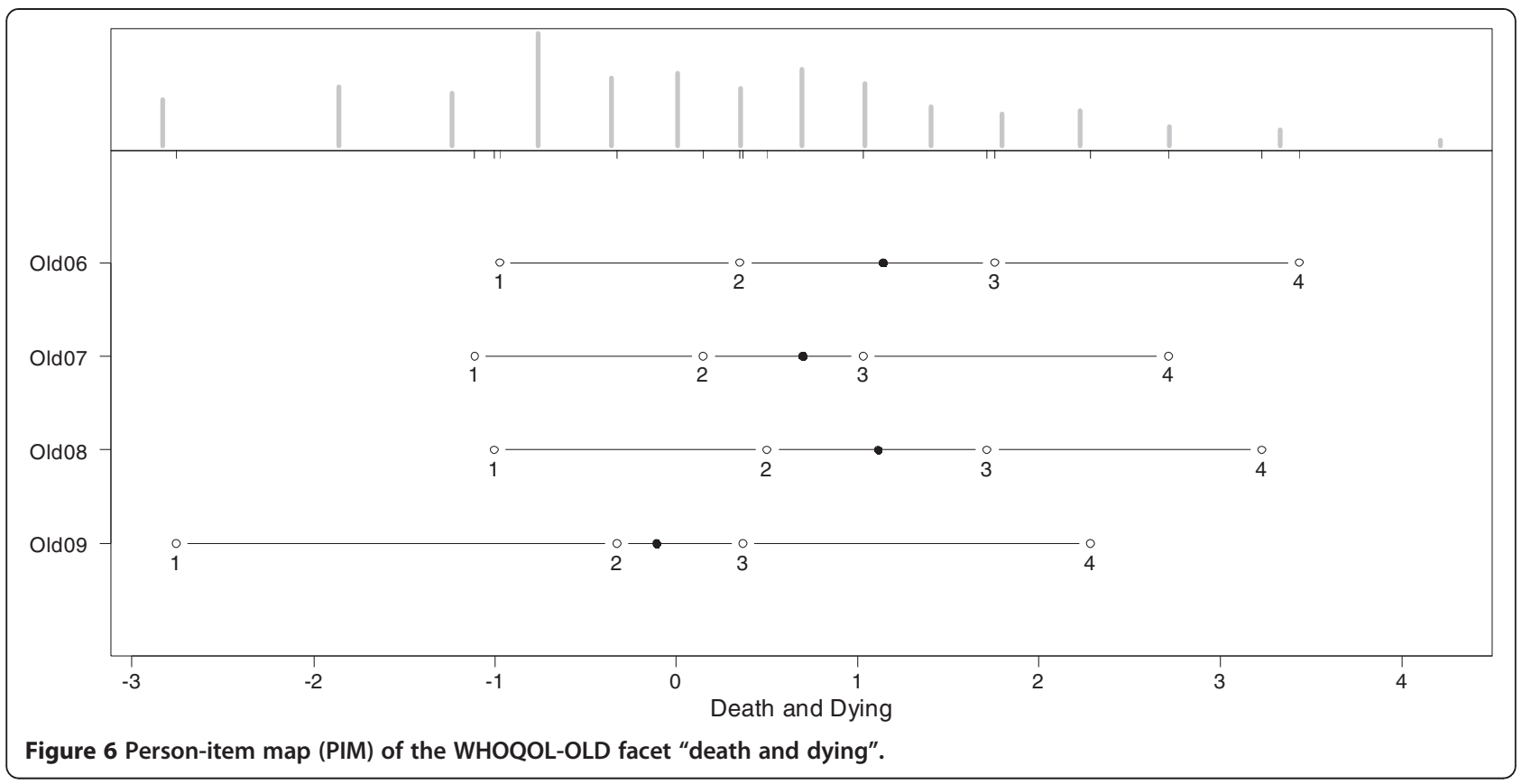

theory" were estimated by the command "alpha" using STATA 13 [50].

\section{Results}

A total of 1133 people aged 60 to 96 years old participated in the study (Table 2). The mean age was 72.3 years (SD 8.7 years). The gender ratio of the sample was about equal. About $50 \%$ of the sample was married and lived together with a spouse, while the other half were separated, divorced, widowed or never married. About $43 \%$ of the study population lived alone, while $57 \%$ lived together with partners, children or other people. About $42 \%$ of the study population had finished ten years or more of formal education, while $58 \%$ had finished less than 10 years of school.

Of the study population $66 \%$ had no cognitive impairments, $24 \%$ had mild impairments and $9.5 \%$ were identified as having severe cognitive impairments according to the DemTect.

The mean Instrumental Activity of Daily Living (IADL) score is 6.7 , indicating that the study participants, on

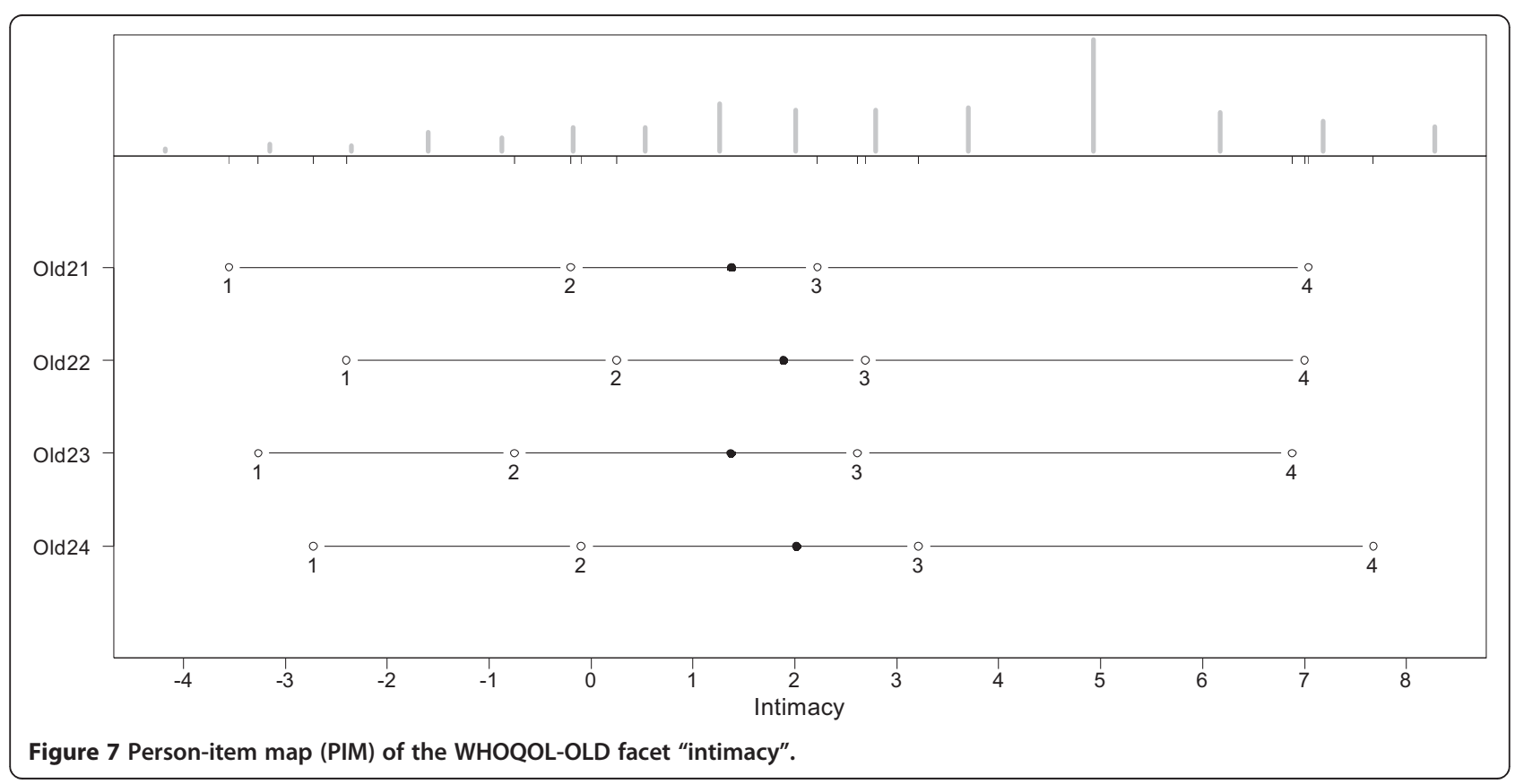



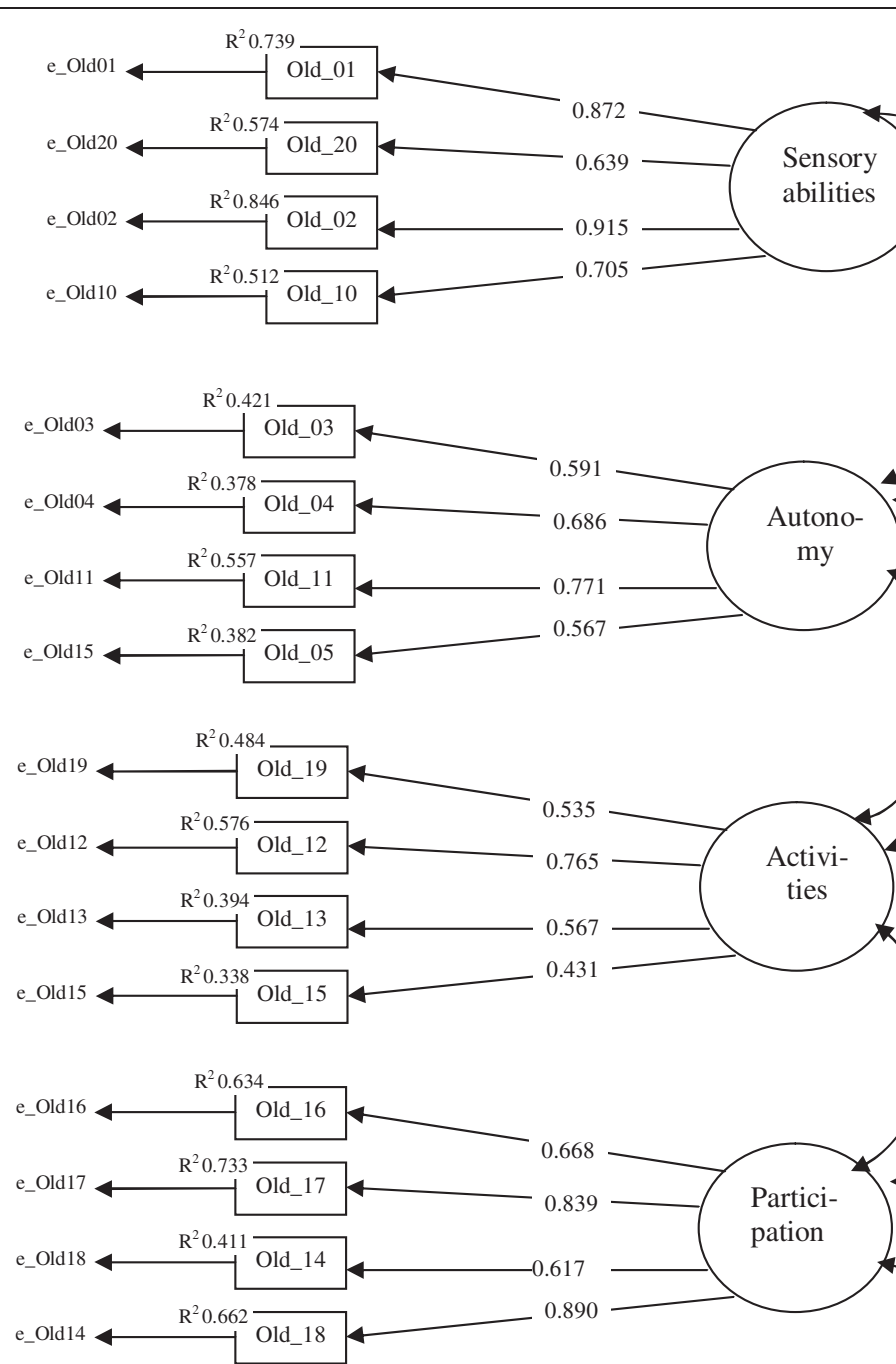
average, are able to live largely independent. The mean Geriatric Depression Scale (GDS) value of 3.5 indicates a low level of depressive symptoms.

\section{Reliability}

Table 3 shows the reliability parameters for the WHOQOLOLD facets according to classical and probabilistic test theory. Cronbach's alpha $(\alpha)$ indicates a high reliability for the WHOQOL-OLD facets sensory abilities $(\alpha=0.8842)$, social participation $(\alpha=0.8502)$, death and dying $(\alpha=$ $0.8567)$, and intimacy $(\alpha=0.9162)$, and a sufficient reliability for the facets autonomy $(\alpha=0.7537)$ and past and present activities $(\alpha=0.7619)$. The corrected item test correlations are above the critical value of 0.3 for all items. The mean inter-item correlations are between 0.4015 for the facet autonomy and 0.7324 for the facet intimacy indicating a high homogeneity of the WHOQOLOLD items.

Reliability coefficients from the IRT partial credit model reveal a good reliability (Andrich reliability) for the facets social participation (0.801), death and dying (0.829) and intimacy (0.888) and a sufficient reliability for the facets sensory abilities (0.798), autonomy (0.703) and past, present and future activities (0.751).

The INFIT parameters between 0.5 and 1.5 indicate that all items are productive for the measurement of the associated facets. The $\mathrm{z}$ values for the transformed Qindex indicate no significant deviance of the response patterns from those expected by the partial credit model.

As indicated by Figures 2, 3, 4, 5, 6 and 7, all facets show ordered answering thresholds for the associated items. The varying threshold ranges within and between the items of each facet indicate considerable differences in the discriminatory power not only of the items but also of the answering categories within the same items.

Frequency distributions for the latent scales indicate negatively skewed distributions for all facets, however the modal value of the facet death and dying is much lower than those of the other facets. Particularly the facet sensory abilities but also death and dying have bimodal distributions.
Validity

\section{Construct validity}

Results of the first order confirmatory factor analysis (Figure 8) reveal that all WHOQOL-OLD facets are represented by sufficient significant standardized loadings above 0.5 on the associated items. The only exception is the small loading of the facet past present and future activities on the item $19(0.339)$. $\mathrm{R}^{2}$ values indicate sufficient communalities of above 0.3 for all items with the exception of item 19 with 0.431 .

As shown in Table 4, correlations between the factors representing the 6 WHOQOL-OLD facets range between $\mathrm{r}=0.180$ between sensory abilities and death and dying and $\mathrm{r}=0.907$ between social participation and past, present and future activities. In particular, the high correlations between the factors representing the facets social participation, autonomy and past, present and future activities suggest that a latent common factor representing a WHOQOL-OLD total score may exist.

To test this assumption, a second order confirmatory factor model was estimated. For this purpose, the variance of the factor representing the WHOQOL-OLD facet past, present and future activities was fixed to zero. The factor loading structure of this model (Figure 9) reveals sufficient standardized loadings above 0.5 of the common factor on five of the six factors representing the WHOQOL-OLD facets. Only the loading on the factor representing the WHOQOL-OLD facet death and dying is 0.295 , which is far below the limit of 0.500 . Moreover, the $\mathrm{R}^{2}$ of 0.087 indicates an insufficient low communality for the factor representing the facet death and dying but with an estimate of 0.257 . This also holds for the factor representing the WHOQOL-OLD facet sensory abilities.

The fit-characteristics for both models are presented in Table 5. The $\mathrm{Chi}^{2}$ values indicate significant deviances from the empirical covariance structure but that would be expected because of the large sample size. The general fit parameters CFI and TFI are sufficient for both models; the same is true for RMSEA and the SRMR. The comparison of the fit parameters between both models reveals no improvement of the model fit by adding the second order common factor. The loadings

Table 4 Inter-correlations of the factors representing the WHOQOL-OLD facets

\begin{tabular}{|c|c|c|c|c|c|}
\hline & Sensory abilities & Autonomy & $\begin{array}{l}\text { Past, present and } \\
\text { future activities }\end{array}$ & $\begin{array}{l}\text { Social } \\
\text { participation }\end{array}$ & Death and dying \\
\hline Autonomy & $0.491^{* * *}$ & & & & \\
\hline Past, present and future activities & $0.466^{* * *}$ & $0.900^{* * *}$ & & & \\
\hline Social participation & $0.487^{* * *}$ & $0.808^{* * *}$ & $0.907^{* * *}$ & & \\
\hline Death and dying & $0.180^{* * *}$ & $0.202^{* * *}$ & $0.323^{* * *}$ & $0.229 * * *$ & \\
\hline Intimacy & $0.323^{* * *}$ & $0.576^{* * *}$ & $0.705^{* * *}$ & $0.557^{* * *}$ & $0.252^{* * *}$ \\
\hline
\end{tabular}

${ }^{*} p \leq 0.05,{ }^{* *} p \leq 0.01,{ }^{* * *} p \leq 0.001$. 


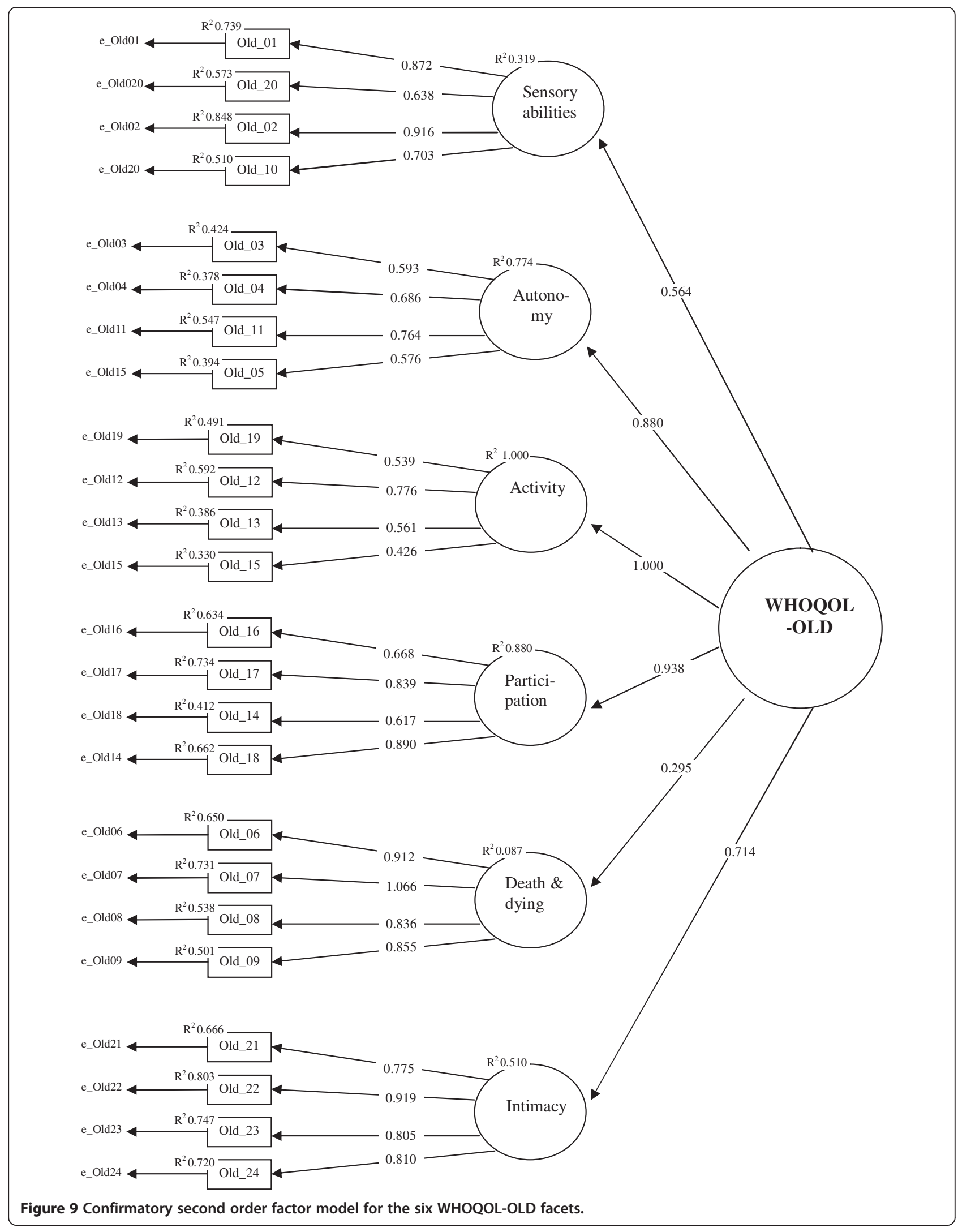


Table 5 Model fit characteristics of the first order and the second order confirmatory factor models for the WHOQOL-OLD facets

\begin{tabular}{lll}
\hline Parameter & $\begin{array}{l}\text { First order } \\
\text { factor model }\end{array}$ & $\begin{array}{l}\text { Second order } \\
\text { factor model }\end{array}$ \\
\hline Chi2 & 897.86 & 941.62 \\
Degrees of freedom & 237 & 247 \\
P & 0.000 & 0.000 \\
CFI & 0.946 & 0.943 \\
TLI & 0.937 & 0.937 \\
RMSEA (90\% Cl) & $0.050(0.0460 .053)$ & $0.050(0.0460 .053)$ \\
Prob. RMSEA < $=0,05$ & 0.565 & 0.528 \\
SRMR & 0.044 & 0.047 \\
Akaike Information & 60733.52 & 60767.70 \\
criterion (AIC) & & \\
$\begin{array}{l}\text { Bayes Information } \\
\text { criterion(BIC) }\end{array}$ & 61171.38 & 61155.22 \\
Adjusted BIC & 60895.02 & 60910.65 \\
\hline
\end{tabular}

clearly show that a one-dimensional representation cannot be recommended (Figure 9).

\section{Convergent validity}

Table 6 shows the correlations between the WHOQOLOLD facets and the criterion variables. With the exception of the death and dying facet, all WHOQOL-OLD facets and the WHOQOL-OLD total score show medium to high positive correlations (between $r=0.363$ and $r=$ 0.798) with the WHOQOL-BREF subscales and the WHOQOL-BREF overall score. Medium to high positive correlations were also found between the WHOQOLOLD facets except death and dying and the SF12 subscales "Physical Health Index" and "Mental Health Index." In contrast to all other WHOQOL-OLD facets, the facet death and dying shows much smaller correlations between $r=0.185$ and $r=0.286$ with the generic quality of life scales.

\section{Discriminant validity}

Results of the linear regression models are presented in Table 7. As indicated by the standardized regression coefficients, depressive symptoms have the strongest negative effect on all six WHOQOL-OLD facets and on the total WHOQOL-OLD score. The level of cognitive functioning has a positive effect on all facets except death and dying and on the total score. The number of chronic diseases is negatively related to sensory abilities and to death and dying and positively related to intimacy.

Socio-demographic characteristics and living arrangements affect only some of the WHOQOL-OLD facets. Increasing age is related to decreasing sensory abilities but positively to past, present and future activities. Female sex is negatively related to autonomy. In comparison to persons who live alone, those who live with others have a higher quality of life on the WHOQOLOLD facets death and dying, intimacy and a higher WHOQOL-OLD total score. Persons with a higher formal education assess their past, present and future activities better than those with a lower educational level.

As indicated by the adjusted $\mathrm{R}^{2}$ a considerable amount of variance was explained by the model variables.

\section{Discussion}

This is the first examination of the psychometric properties of the WHOQOL-OLD for a representative sample of the German population aged 60 years and older. Psychometric properties were examined by means of the classic test theory and, essentially, by probabilistic test theory.

The examination of the parameters for the internal consistency revealed high reliability coefficients and high item-scale respective intern item correlations for four facets sensory abilities, participation, death and dying and intimacy of the six facets of the WHOQOLOLD. The remaining two facets autonomy and activity show low, but still acceptable, values for the internal consistency.

Table 6 Person correlations of WHOQOL-OLD facets with criterion variables

\begin{tabular}{|c|c|c|c|c|c|c|c|}
\hline \multirow[b]{2}{*}{ Criterion variable } & \multicolumn{7}{|c|}{ WHOQOL-OLD facets } \\
\hline & Sensory abilities & Autonomy & $\begin{array}{l}\text { Past, present } \\
\text { and future activities }\end{array}$ & $\begin{array}{l}\text { Social } \\
\text { participation }\end{array}$ & $\begin{array}{l}\text { Death and } \\
\text { dying }\end{array}$ & Intimacy & OLD \\
\hline WHOQOL-BREF Physical & $0.589^{* * *}$ & $0.622^{* * *}$ & $0.631^{* * *}$ & $0.737^{* * *}$ & $0.269^{* * *}$ & $0.434^{* * *}$ & $0.738^{* * *}$ \\
\hline WHOQOL-BREF Psychological & $0.553^{* * *}$ & $0.666^{* * *}$ & $0.729^{* * *}$ & $0.747^{* * *}$ & $0.286^{* * *}$ & $0.578^{* * *}$ & $0.798^{* * *}$ \\
\hline WHOQOL-BREF Social & $0.363^{* * *}$ & $0.518^{* * *}$ & $0.616^{* * *}$ & $0.590^{* * *}$ & $0.240^{* * *}$ & $0.657^{* * *}$ & $0.674^{* * *}$ \\
\hline WHOQOL-BREF Environment & $0.459^{* * *}$ & $0.656^{* * *}$ & $0.660^{* * *}$ & $0.699 * * *$ & $0.185^{* * *}$ & $0.576^{* * *}$ & $0.722^{* * *}$ \\
\hline WHOQOL-BREF Overall & $0.486^{* * *}$ & $0.518^{* * *}$ & $0.595^{* * *}$ & $0.647^{* * *}$ & $0.231^{* * *}$ & $0.414^{* * *}$ & $0.648^{* * *}$ \\
\hline SF12 PHS & $0.536^{* * *}$ & $0.494^{* * *}$ & $0.484^{* * *}$ & $0.593^{* * *}$ & $0.213^{* * *}$ & $0.313^{* * *}$ & $0.592^{* * *}$ \\
\hline SF12 MHS & $0.395^{* * *}$ & $0.514^{* * *}$ & $0.553^{* * *}$ & $0.538^{* * *}$ & $0.281^{* * *}$ & $0.478^{* * *}$ & $0.623^{* * *}$ \\
\hline
\end{tabular}

${ }^{*} p \leq 0.05,{ }^{* *} p \leq 0.01,{ }^{* * *} p \leq 0.001$. 
Table 7 Linear regression models for the WHOQOL-OLD facets (standardized beta coefficients)

\begin{tabular}{|c|c|c|c|c|c|c|c|}
\hline & Sensory abilities & Autonomy & $\begin{array}{l}\text { Past, present } \\
\text { and future activities }\end{array}$ & Social participation & Death and dying & Intimacy & OLD \\
\hline Age & $-.275^{* * *}$ & -.011 & $.108^{* * *}$ & .008 & .033 & .034 & -.027 \\
\hline Female sex & .048 & $-.072^{*}$ & -.013 & -.038 & -.036 & .027 & -.019 \\
\hline Living with others & -.064 & -.060 & -.003 & .017 & $.131^{*}$ & $.282^{* * *}$ & $.081^{*}$ \\
\hline Higher education & -.005 & -.012 & $.075^{*}$ & .020 & -.048 & -.005 & .000 \\
\hline Married & -.046 & .037 & -.024 & -.012 & .098 & -.039 & .007 \\
\hline GDS & $-.406^{* * *}$ & $-.581^{* * *}$ & $-.707^{* * *}$ & $-.665^{* * *}$ & $-.289^{* * *}$ & $-.576^{* * *}$ & $-.722^{* * *}$ \\
\hline IADL & .035 & $.088^{*}$ & -.046 & $.114^{* * *}$ & $-.141^{* * *}$ & -.013 & .003 \\
\hline No. of chronic Diseases & $-.121^{* * *}$ & -.000 & .016 & -.017 & $-.184^{* * *}$ & $.109^{* * *}$ & $-.053^{*}$ \\
\hline DemTect & $.099^{* * *}$ & $.141^{* * *}$ & $.113^{* * *}$ & $.116^{* * *}$ & -.015 & $.054^{*}$ & $.110^{* * *}$ \\
\hline $\mathbf{N}$ & 956 & 956 & 956 & 956 & 956 & 956 & 956 \\
\hline Adjusted $\mathrm{R}^{2}$ & 0.44 & 0.44 & 0.52 & 0.59 & 0.15 & 0.45 & 0.66 \\
\hline
\end{tabular}

Results of the probabilistic test theory approach indicate that all facets of the WHOQOL-OLD can be represented by a partial credit model with ordered thresholds. Fit indices show that all items are productive for measurement. The thresholds of the answering categories have an ascending order for all items but the varying thresholds between the answering categories indicate that the measurement characteristics of the items and the answering categories are unequal.

The construct validity of the six-facet model of the WHOQOL-OLD was supported by the first order confirmatory factor analysis for the six facets model but not by the second order model for the WHOQOL-OLD total scale.

Convergent validity of the WHOQOL-OLD facets could be well confirmed with regard to the subscales of the generic quality of life measures WHOQOL-BREF and SF12.

Results from the multiple regression models indicate that symptoms of depression are the strongest predictor of all WHOQOL-OLD facets. Nevertheless, cognitive functioning, the ability to carry out daily activities and chronic diseases are also important factors in explaining quality of life.

Results of our analyses reveal that the psychometric properties of the German version of the WHOQOLOLD are similar, as good as, or better than those reported from the international WHOQOL-OLD field study [51] and as those of other country versions recently tested in Norway [52], China [21], Brazil [53,54], France [55] and Turkey [56].

As revealed by Power et al. [51] for the international WHOQOL-OLD data set and by Liu et al [57] for the Chinese version of the WHOQOL-OLD, a good construct validity was obtained for the German version of the WHOQOL-OLD in our study for the six facet structure but not for second order factor model. These results underline that the WHOQOL-OLD represents a multidimensional construct of quality of life in old age that cannot be reduced to one latent dimension. Nevertheless, efforts have been made to develop a short version of the WHOQOL-OLD [57] and the authors recommend three versions with different selections of six items from the WHOQOL-OLD. However, the reliability of all three versions of this instrument is worse in comparison to that of the WHOQOL-OLD.

As in the cross-cultural WHOQOL-OLD studies [51] and in several national studies [21,58-60], depressive symptoms were also found to explain a considerable amount of variance in all facets of the German version. Chachamovic et al. [60] examined the effects of a major depression diagnosis in comparison to subclinical symptoms of depression and found that even in the absence of a diagnosis of a major depression, sub clinical symptoms of depression have a strong negative effect on all facets of the WHOQOL-OLD.

The strong negative effect of depressive symptoms on QoL in the German population corresponds with results from cross cultural studies on the importance of different domains of QoL showing that the presence of positive feelings and the absence of negative feelings ranked higher than average in the German sample [61]. The importance of positive feelings on QoL could be related to the high level of economic development in Germany. Economic development has been identified as a major cultural factor in explaining the variance in cross cultural importance rankings. While in developing countries the facets related to physical health were ranked higher than those related to psychological well-being, the opposite was the case in developed countries [61]. Nevertheless, associations of important rankings of psychological well-being with economical development do 
not necessarily result in different effects of depressive symptoms on QoL. Dragomirecka et al. [62] identified depressive symptoms as the main predictors of most WHOQOL-OLD domains in all countries independent of the countries' economical wealth status in their cross cultural comparison of QoL in the elderly population of six European countries [62]. These results support the hypothesis that depressive symptoms are intercultural predictors of quality of life in elderly people. However, since most studies on QoL in elderly people are crosssectional, the exact relationships between objective living circumstances, cultural factors, depressive symptoms and QoL are still unclear. Longitudinal cross-cultural studies would allow for the analysis of whether cultural factors or symptoms of depression work as mediator or moderator variables in this relationship.

\section{Limitations}

Due to the cross-sectional design of the study, test-retest reliability and sensitivity to change of the WHOQOLOLD could not be assessed. The clinical status of the respondents was assessed by means of the self-rating GDS, which does not allow the diagnosis of major depression. Therefore, it was not possible to examine differences between the impact of clinical and sub-clinical levels of depression.

\begin{abstract}
Abbreviations
WHOQOL: World Health Organization Quality of Life; GDS: Geriatric depression scale; IADL: Instrumental activities of daily living; IRT: Item response theory; MCl: Mild cognitive impairment; PCM: Partial credit model; PSI: Person-separation-index; PIM: Person-item map; SRMR: Standardized root mean square residual; RMSEA: Root mean square error of approximation; CFI: Comparative fit index; TLI: Tucker-lewis index.
\end{abstract}

\section{Competing interests}

The authors declare that they have no competing interest.

\section{Authors' contributions}

IC, HM and RK designed the study, extracted data, performed the statistical analysis, interpreted the results, and drafted the manuscript. SRH contributed to the study design, contributed to the interpretation of the results, and to the revision of the manuscript. CG contributed to the interpretation of the results and collected data. All authors read and approved the final manuscript.

\section{Acknowledgments}

The study was funded by the German Research Foundation DFG (CO 900/1-1; KI 792/2-1)

\section{Author details \\ ${ }^{1}$ Institute for Social Medicine, Occupational Health and Public Health, University of Leipzig, Medical Faculty, Philipp-Rosenthal-Str. 55, 04103 Leipzig, Germany ${ }^{2}$ Institute of Health Economics and Health Service Research, University of Hamburg, Hamburg Center for Health Economics, Martinistr. 52, 20246 Hamburg, Germany. ${ }^{3}$ Department of Psychiatry and Psychotherapy II, UIm University, Ludwig-Heilmeyer-Str. 2, 89312 Günzburg, Germany.}

Received: 24 February 2014 Accepted: 19 June 2014 Published: 5 September 2014

\section{References}

1. United Nations Organization: World population ageing 1950-2021. New York: United Nations; 2002.
2. U.S. Census Press Release: Retrieved February 11, 2008 from. 2005, http://www.census.gov/Press-Release/www/releases/archives/facts_for_ features_special_editions/004210.html.

3. Organisation for Economic Cooperation and Development: Maintaining prosperity in an ageing society. Paris: OECD; 1999.

4. Blane $D$, Higgs $P$, Hyde $M$, Wiggins R: Life course influences on quality of life in early old age. Soc Sci Med 2004, 58:2171-2179.

5. Baltes PB, Smith J: New frontiers in the future of aging: from successful aging of the young old to the dilemma of the fourth age. Gerontology 2003, 49:123-136.

6. Haywood KL, Garratt AM, Schmidt L, Mackintosh A, Fitzpatrick R: Health status and quality of life of life in older people: a review. Oxford: National Centre for Health Outcomes Development; 2004.

7. Bowling A: Measuring health. Buckingham: Open University Press; 1997.

8. Power M, Quinn K, Schmidt S: Development of the WHOQOL-Old Module. Qual Life Res 2005, 14:2197-2214.

9. Sarvimäki A, Stenbock-Hult B: Quality of life in old age described as a sence of well-being, meaning and value. J Adv Nurs 2000, 32:1025-1033.

10. Higgs $P$, Hyde $M$, Wiggins $R$, Blane D: Researching quality of life in early old age: the importance of the sociological dimension. Soc Policy Adm 2003, 37:239-252.

11. Hunt S: The problem of quality of life. Qual Life Res 1997, 6:205-212.

12. The WHOQOL Group: Study protocol for the World Health Organisation project to develop a quality of life assessment instrument (WHOQOL). Qual Life Res 1993, 2:153-159.

13. The WHOQOL Group: The development of the World Health Organization quality of life assessment instrument: The WHOQOL. In Quality of Life Assessment: International Perspectives. Edited by Kuyken W, Orley J. Berlin: Springer; 1994:41-57.

14. The WHOQOL Group: Development of the World Health Organization WHOQOL-BREF Quality of Life Assessment. Psychol Med 1998, 28:551-558.

15. The WHOQOL Group: The World Health Organization Quality of Life Assessment (WHOQOL): Development and General Psychometric Properties. Soc Sci Med 1998, 46:1569-1585.

16. Power M, Bullinger M, Harper A, The WHOQOL Group: The World Health Organization WHOQOL-100: Tests of the Universality of Quality of Life in 15 Different Cultural Groups Worldwide. Health Psychol 1999, 18:495-505.

17. Skevington S: Advancing cross-cultural research on quality of life: observations drawn from the WHOQOL development. Qual Life Res 2002, 11:135-144.

18. Saxena S, Carlson D, Billington R, Orley J, The WHOQOL Group: The WHO quality of life assessment instrument (WHOQOL-BREF): The importance of its items for cross-cultural research. Qual Life Res 2001, 10:711-721.

19. Angermeyer M, Kilian R, Matschinger H: WHOQOL-100 und WHOQOL-BREF. Handbuch für die deutschsprachige Version der WHO Instrumente zur Erfassung von Lebensqualität. Göttingen: Hogrefe; 2000.

20. Winkler I, Buyantugs L, Petscheleit A, Kilian R, Angermeyer M, The WHOQOL OLD Group: Die interkulturelle Erfassung der Lebensqualität im Alter: Das WHOQOL-OLD-Projekt. Z f Gerontopsychologie und -psychiatrie 2003, 16:177-192

21. Liu R, Wu S, Hao Y, Gu J, Fang J, Cai N, Zhang J: The Chinese version of the world health organization quality of life instrument-older adults module (WHOQOL-OLD): psychometric evaluation. Health Qual Life Outcomes 2013, 11:156.

22. Kalbe E, Kessler J, Calabrese P, Smith R, Passmore AP, Brand M, Bullock R: DemTect: a new, sensitive cognitive screening test to support the diagnosis of mild cognitive impairment and early dementia. Int J Geriatr Psychiatry 2004, 19:136-143.

23. Winkler I, Matschinger $\mathrm{H}$, Angermeyer M: The WHOQOL-OLD - A questionnaire for the intercultural measuring of quality of life in the elderly. Psychother Psychosom Med Psychol 2006, 56:63-69.

24. Robert Koch Institut: Bundes-Gesundheitssurvey. 1998, Internet: http://www.rki.de; Stand: 09.08.2012

25. Kovar MG, Lawton MP: Functional disability: activities and instrumental activities of daily living. In Focus on assessment techniques. Volume 14 Edited by Lawton MP, Teresi JA. New York: Springer; 1994:57-75.

26. Lawton MP, Brody EM: Assessment of older people: self-maintaining and instrumental activities of daily living. Gerontologist 1969, 9:179-186.

27. Edelen $\mathrm{MO}$, Reeve BB: Applying item response theory (IRT) modeling to questionnaire development, evaluation, and refinement. Qual Life Res 2007, 16:5-18. 
28. Reeve BB, Hays RD, Chih-Hung C, Perfetto EM: Applying item response theory to enhance health outcomes assessment. Qual Life Res 2007, 16:1-3.

29. Cook KF, Teal CR, Bjorner JB, Cella D, Chih-Hung C, Crane PK, Gibbons LE, Hays RD, McHorney CA, Ocepek-Welikson K, Raczek AE, Teresi JA, Reeve BB: IRT health outcomes data analysis project: an overview and summary. Qual Life Res 2007, 16:121-132.

30. Dorans NJ: Linking scores from multiple health outcome instruments. Qual Life Res 2007, 16:85-94.

31. Masters GN: A Rasch model for partial credit scoring. Psychometrika 1982 47:149-174.

32. Masters GN: The analysis of partial credit scoring. Appl Meas Educ 1988 $1: 279-297$.

33. Wright BD, Masters GN: Rating scale analysis. Chicago: MESA Press; 1982.

34. Rasch G: Probabilistic Models for Some Intelligence and Attainment Tests. Chicago: The University of Chicago (Originally published 1960); 1980

35. Rost J: Measuring attitudes with a threshold model drawing on a traditional scaling concept. Appl Psychol Meas 1988, 12:397-409.

36. Masters GN, Wright BD: The essential process in a family of measurement models. Psychometrika 1984, 49:529-544.

37. Verhelst ND, Verstralen HHFM: Some considerations on the partial credit model. Psicologica 2008, 29:229-254.

38. Wright BD, Masters GN: Computation of OUTFIT and INFIT statistics. Rasch Meas Trans 1990, 3(4):84-85.

39. Bond TG, Fox CM: Applying the Rasch model. 2nd edition. NJ: L. Erlbaum Mahwah; 2007.

40. Smith RM: Fit analysis in latent trait measurement models. J Appl Meas 2000, 1:199-218.

41. Smith RM: Polytomous mean-square fit statistics. Rasch Meas Trans 1996, 10:516-517.

42. Linacre JM, Wright BD: Reasonable mean square fit values. Rasch Meastran 1994, 83:370

43. Rost J, von Davier M: A conditional item-fit index for Rasch models. Appl Psychol Meas 1994, 18:171-182

44. Andrich D: Rasch Models for Measurement. Newbury Park: SAGE Publications; 1988.

45. von Davier M: WINMIRA; a program system for analysis with the Rasch Model, with the Latent Class Analysis and with the Mixed Rasch Model. Kiel: IPN 2001.

46. Jöreskog KG: Statistical analysis of sets of congeneric tests. Psychometrika 1971, 36:109-133.

47. Muthén LK, Muthén BO: Mplus user's guide. 7th edition. Los Angeles: Muthén \& Muthén; 2012

48. Mair P, Hatzinger R: Extended Rasch modeling: the eRm package for the application of IRT models in R. J Stat Softw 2007, 20:1-20.

49. Rizopoulos D: An R package for latent variable modeling and item response theory analyses. J Stat Softw 2006, 17:1-25.

50. StataCorp: Stata Statistical Software: Release 13. TX: StataCorp LP; 2013.

51. Power M, Quinn K, Schmidt S, and the WHOQOL-GROUP: Development of the WHOQOL-OLD module. Qual Life Res 2005, 14:2197-2214.

52. Halvorsrud L, Kalfoss M, Diseth A: Reliability and validity of the Norwegian WHOQOL-OLD module. Scand J Caring Sci 2008, 22:292-305.

53. Chachamovich E, Fleck MP, Trentini C, Power M: Brazilian WHOQOL-OLD Module version: a Rasch analysis of a new instrument. Rev Saude Publica 2008, 42:308-316.

54. Fleck MP, Chachamovich E, Trentini CM: WHOQOL-OLD Project: method and focus group results in Brazil. Rev Saude Publica 2003, 37:793-799.

55. Leplege A, Perret-Guillaume C, Ecosse E, Hervy MP, Ankri J, von SN: A new instrument to measure quality of life in older people: the French version of the WHOQOL-OLD. Rev Med Interne 2013, 34:78-84.

56. Eser $\mathrm{S}$, Saatli G, Eser E, Baydur H, Fidaner C: The reliability and validity of the Turkish Version of the World Health Organization Quality of Life Instrument-Older Adults Module (WHOQOL-Old). Turk Psikiyatri Derg 2010, 21:37-48.

57. Fang J, Power M, Lin Y, Zhang J, Hao Y, Chatterji S: Development of short versions for the WHOQOL-OLD module. Gerontologist 2012, 52:66-78.

58. Lucas-Carrasco R, Laidlaw K, Power MJ: Suitability of the WHOQOL-BREF and WHOQOL-OLD for Spanish older adults. Aging Ment Health 2011 15:595-604

59. Halvorsrud L, Kirkevold M, Diseth A, Kalfoss M: Quality of life model: predictors of quality of life among sick older adults. Res Theory Nurs Pract 2010, 24:241-259.
60. Chachamovich E, Fleck M, Laidlaw K, Power M: Impact of major depression and subsyndromal symptoms on quality of life and attitudes toward aging in an international sample of older adults. Gerontologist 2008, 48:593-602.

61. Molzahn AE, Kalfoss M, Schick MK, Skevington SM: Comparing the importance of different aspects of quality of life to older adults across diverse cultures. Age Ageing 2011, 40:192-199.

62. Dragomirecka E, Bartonova J, Eisemann M, Kalfoss M, Kilian R, Martiny K, von Steinbuechel N, Schmidt S: Demographic and psychosocial correlates of quality of life in the elderly from a cross-cultural perspective. Clin Psychol Psychother 2008, 15:193-204.

doi:10.1186/s12955-014-0105-4

Cite this article as: Conrad et al:: The psychometric properties of the German version of the WHOQOL-OLD in the German population aged 60 and older. Health and Quality of Life Outcomes 2014 12:105.

\section{Submit your next manuscript to BioMed Central and take full advantage of:}

- Convenient online submission

- Thorough peer review

- No space constraints or color figure charges

- Immediate publication on acceptance

- Inclusion in PubMed, CAS, Scopus and Google Scholar

- Research which is freely available for redistribution

Submit your manuscript at www.biomedcentral.com/submit
C Biomed Central 